\title{
A New Online Identification Methodology for Flux and Parameters Estimation of Vector Controlled Induction Motors
}

\author{
Vicente Leite ${ }^{(1)}$, Rui Araújo ${ }^{(2)}$, Member, IEEE, Diamantino Freitas ${ }^{(3)}$ \\ ${ }^{(1)}$ Instituto Politécnico de Bragança - Escola Superior de Tecnologia e de Gestão \\ Campus de Sta. Apolónia - Apartado 134, 5301-857 Bragança, Portugal, avtl@ipb.pt \\ (2), (3) Faculdade de Engenharia da Universidade do Porto \\ Rua Dr. Roberto Frias s/n, 4200-465 Porto, Portugal, ${ }^{(2)}$ raraujo@fe.up.pt, ${ }^{(3)}$ dfreitas@fe.up.pt
}

\begin{abstract}
A new online identification methodology for estimation of the rotor flux components and the main electrical parameters of vector controlled induction motors is presented in this paper. The induction motor model is referred to the rotor reference frame for estimation of rotor flux and rotor parameters, and referred to the stator reference frame to estimate stator parameters. The stator parameters estimation is achieved by a prediction error method based on a model structure described by a linear regression that is independent of rotor speed and rotor parameters. The rotor flux components and rotor parameters are estimated by a reduced order extended Kalman filter, using a $4^{\text {th }}$-order state-space model structure where the state equation is described by matrices that are diagonal and independent of rotor speed as well as stator parameters. Both methods work in a boot-strap manner.
\end{abstract}

\section{INTRODUCTION}

In recent years, the availability of digital signal processors and the fast development of modern power electronics have contributed to the wide acceptance of the prevailing vector control in high-performance induction motor (IM) drives, and have made the correct estimation of the rotor flux and the adaptation of the system to changing motor parameters feasible, which are two major problems in the implementation of such industrial applications as referred in [1]. The recent trend is to implement a solution with joint online estimation of IM states and parameters [1-5]. However, the simultaneous estimation of the two rotor flux components and all electrical parameters of a vector controlled induction motor, under normal operating conditions, for real-time implementations, remains a challenge. This work is a new contribution for this purpose.

The main goal is to achieve the simultaneous estimation of flux and electrical parameters in real time operation, with as low computational effort as possible, under normal operating conditions and with independent and flexible estimators in order to adjust the identification procedure to the dynamic conditions of the machine.

\section{INDUCTION MOTOR MODEL}

In previous works $[4,5]$ the authors have shown that the joint estimation of rotor flux components and all the electrical

The authors would like to acknowledge the financial assistance provided by Fundação Luso-Americana. parameters of a per-phase IM model described by four parameters [6], is feasible in real time applications by using an extended Kalman filter (EKF) technique, with reduction of computational effort, based on a reduced order model in the rotor reference frame as shown below:

$$
\begin{aligned}
& {\left[\begin{array}{c}
\dot{\psi}_{r d}^{r} \\
\dot{\psi}_{r q}^{r}
\end{array}\right]=\left[\begin{array}{cc}
-\tau_{r}^{-1} & 0 \\
0 & -\tau_{r}^{-1}
\end{array}\right]\left[\begin{array}{c}
\psi_{r d}^{r} \\
\psi_{r q}^{r}
\end{array}\right]+\left[\begin{array}{cc}
L_{M} \tau_{r}^{-1} & 0 \\
0 & L_{M} \tau_{r}^{-1}
\end{array}\right]\left[\begin{array}{c}
i_{s d}^{r} \\
i_{s q}^{r}
\end{array}\right](1)} \\
& u_{s d}^{r}=-\tau_{r}^{-1} \psi_{r d}^{r}-\omega \psi_{r q}^{r}+\left(R_{s}+L_{M} \tau_{r}^{-1}\right) i_{s d}^{r}+L_{s}^{\prime}\left(\dot{i}_{s d}^{r}-\omega i_{s q}^{r}\right)(2)
\end{aligned}
$$

where: $\quad \psi_{r d}^{r}(t)=L_{m} L_{r}^{-1} \phi_{r d(t)}^{r}, \quad \psi_{r q}^{r}(t)=L_{m} L_{r}^{-1} \phi_{r q(t)}^{r}$, $\tau_{r}=L_{r} R_{r}^{-1}, L_{s}^{\prime}=L_{s}-L_{m}^{2} L_{r}^{-1}$ and $L_{M}=L_{m}^{2} L_{r}^{-1}$.

The simultaneous estimation of the four electrical parameters requires some dynamic conditions for a correct identification of stator parameters $[4,5]$, namely, $R_{S}$ and $L_{S}^{\prime}$. In any case the estimation of these parameters remains hard to do, wherefore another procedure is presented in the next section. Oppositely, the other two motor parameters, $\tau_{r}$ and $L_{M}$, can be estimated whether in transient conditions or in steady-state operation with a good robustness with respect to errors in the stator parameters [4]. According to this fact, the authors propose a new methodology that enables the estimation of the main parameters but separates the estimation of $\tau_{r}, L_{M}$ and the rotor flux components from the stator parameters.

\section{The IDENTIFICATION MEthodology}

The proposed methodology consists of a first estimator that uses the EKF to find good estimates of the parameters $\tau_{r}$ and $L_{M}$, and the two rotor flux components, followed by a recursive prediction error based estimator (RPEM) to obtain the stator resistance and either the transient stator inductance, $L_{s}^{\prime}$, or the stator inductance, $L_{s}$, estimated values, together or separately, in a boot-strap manner. Thus, considering a specific instant, $t_{k}$, the EKF is used for estimation of the extended and scaled state vector $\hat{x}$, based on previous 
estimated values of stator parameters and then, a RPEM is used to estimate the parameter vector $\hat{\theta}$, which corresponds to the scaled parameters $L_{s}^{\prime}$ (or $L_{s}$ ) and $R_{s}$. The state vector $x$ and the parameter $\theta$ are scaled by constants $K_{i}$ 's, for numerical reasons, as follows:

$$
\begin{aligned}
& \bar{x}\left(k, \widehat{\theta}_{t_{k-1}}\right)=\left[\begin{array}{llll}
\hat{x}_{1}(k) & \hat{x}_{2}(k) & \hat{x}_{3}(k) & \hat{x}_{4}(k)
\end{array}\right]= \\
& =\left[\begin{array}{lllll}
k_{1} \psi_{r d}^{r}(k) & k_{2} \psi_{r q}^{r}(k) & k_{3} \tau^{-1}(k) & k_{4} L_{M}(k)
\end{array}\right] \\
& \hat{\theta}\left(k, \widehat{x}_{t_{k}}\right)=k_{5} L_{s}^{\prime}(k) \text { or } \\
& \hat{\theta}\left(k, \hat{x}_{t_{k}}\right)=k_{6} R_{s}(k) \text { or } \\
& \hat{\theta}\left(k, \hat{x}_{t_{k}}\right)=\left\lfloor k_{5} L_{s}^{\prime}(k) \quad k_{6} R_{s}(k)\right\rfloor .
\end{aligned}
$$

For the EKF algorithm, the model structure is formed by a nonlinear discrete state space $4^{\text {th }}$-order model, obtained by discretization of state equation in (1), and the output equation in (2), as presented in [5] and results as follows, where $T_{s}$ is the sampling period:

$$
\begin{aligned}
& {\left[\begin{array}{l}
\psi_{r d}^{r}(k+1) \\
\psi_{r q}^{r}(k+1)
\end{array}\right]=\left[\begin{array}{cc}
1-T_{s} \tau_{r}^{-1} & 0 \\
0 & 1-T_{s} \tau_{r}^{-1}
\end{array}\right]\left[\begin{array}{l}
\psi_{r d}^{r}(k) \\
\psi_{r q}^{r}(k)
\end{array}\right]+} \\
& +\left[\begin{array}{cc}
T_{s} L_{M} \tau_{r}^{-1} & 0 \\
0 & T_{s} L_{M} \tau_{r}^{-1}
\end{array}\right]\left[\begin{array}{c}
i_{s d}^{r}(k) \\
i_{s q}^{r}(k)
\end{array}\right] \\
& u_{s d}^{r}(k)-\widehat{R}_{s} i_{s d}^{r}(k)-\hat{L}_{s}^{\prime}\left(i_{s d}^{r}\left(t_{k}\right)-\omega(k) i_{s q}^{r}(k)\right)= \\
& =-\tau_{r}^{-1} \psi_{r d}^{r}(k)-\omega(k) \psi_{r q}^{r}(k)+L_{M} \tau_{r}^{-1} i_{s d}^{r}(k)
\end{aligned}
$$

As we can see, the state equation in (5) is independent of rotor speed and stator parameters. Moreover, the matrices are diagonal and, therefore, it becomes very simple to get higher order approximation in the discretization process. The rotor speed and stator parameters only appear in the output equation in (6) where the measured output is a function of the stator parameters which are estimated by using the model structure described bellow.

For the recursive prediction error based method, a linear regression model structure is used, which is derived from the stator voltage equation, expressed in the stator reference frame, as follows:

$$
u_{s d}^{s}(k)=R_{s} i_{s d}^{s}(k)+L_{s}^{\prime}(k) \dot{i}_{s d}^{s}\left(t_{k}\right)+\dot{\bar{\psi}}_{r d}^{s}\left(t_{k}\right) .
$$

Obviously, instead of the $d$ components in (7), the $q$ ones could be selected. The equation above can then be rewritten as a general linear regression as follows:

$$
y(k, \widehat{x})=\theta(k) u(k)
$$

where, for the case of full estimation, like in (4c), we have:

$$
y(k, \widehat{x})=u_{s d}^{s}(k)-\dot{\bar{\psi}}_{r d}^{s}\left(t_{k}\right)
$$

and

$$
\theta(k) u(k)=R_{s} i_{s d}^{s}(k)+L_{s}^{\prime}(k) i_{s d}^{s}\left(t_{k}\right) .
$$

As we can verify the equations (9) and (10), described as a linear regression like in (8), do not depend on neither the rotor parameters nor the rotor speed. However, they depend on rotor flux $d$ (or $q$ ) component's first derivative. The first derivatives in (9) and (10) are computed by the following general recursive filter that can be found in [7], in order to obtain better results than by using Euler's formula,

$$
\dot{x}=d x /\left.d t\right|_{t=t_{k}} \approx \frac{1}{T_{s}} \sum_{i=0}^{n-1} C_{i} x\left(t_{k}-i T_{s}\right)
$$

The weights $C_{i}$ can also be found in [7] and are determined after Taylor series expansion of the equation above to $m+1$ terms, with $m=\{1,2, \cdots, n\}, m$ being the order of the filter and $n$ the number of points. For system identification purposes, as in this case, an important aspect to take into account is the delay introduced by the filter. The delay must be the same as the one introduced by the Euler's formula that is implicit in the discretization process of sate equation in (1) if the linear terms of the Taylor's development are adopted as in (5). For sampling frequencies below $5 \mathrm{kHz}$, the set of coefficients $\left[\begin{array}{llll}11 & -18 & 9 & -2\end{array}\right] / 6$, has produced the best results. This is an unusual discretization process. Indeed, this strategy presented by the authors in $[4,5]$ has been proven to give better results.

This methodology is similar to an adaptive state estimator for nonlinear systems described, in general terms, in [8] and applied here for joint state and parameter estimation. The setup in fig. 1 is a very natural and simple way to achieve this objective. Beyond the advantage of separating the estimation of $\tau_{r}, L_{M}$ and the rotor flux components from the stator parameter, by adapting the estimator to the machine operating point or, in other words, to the information contained in the measured signals, it permits to overcome many of the disadvantages associated with the EKF, namely, a strong computational effort, eventually biased estimates, and not guaranteed convergence [8]. Furthermore, this can be a good alternative to the extended Luenberger observer suggested in [1] to solve the steady-state bias problem detected in the joint rotor flux and rotor time constant estimation, and an alternative to [2,3], in terms of computational effort.

As far as the induction motor is concerned the main advantage of this methodology is that it enables the simultaneous estimation of rotor flux components and rotor parameters by using the EKF, and the estimation of stator parameters by using a RPEM based approach for joint estimation of stator parameters or even two RPEMs for independent estimation of these parameters. 
The independence of the two or three algorithms, as represented in fig. 2(a) and (b), is really very important since the operating conditions of the induction motor required for a successful simultaneous estimation of all electrical parameters, are quite different for the four parameters.

In $[4,5]$ the authors have shown that both rotor parameters $\left(\tau_{r}\right.$ and $\left.L_{M}\right)$ are well estimated even in steady-state operation and, due to this, they are proposed to be estimated together with rotor flux components in every iteration. On the other hand, stator transient inductance needs significant dynamic conditions which are different from the ones needed for stator resistance estimation that should be estimated at low rotor speed where the term $R_{s} i_{s d}^{s}$ in (6) becomes important improving, by this way, the sensibility of the model structure in relation to stator resistance. It can be easily seen that at high speed that term is negligible when added to stator voltage in (6) and as a result, the stator resistance starts to diverge, although slowly.

By using this new methodology the estimation of stator parameters can be separated from flux and the rotor ones which can be enabled or disabled according to the dynamic conditions of the induction motor and this is very important to do and even mandatory. Furthermore, the EKF based algorithm that estimates rotor flux and rotor parameters can update, at every iteration, the values of the stator parameters in its output equation, from time to time, or only when they are properly available by the respective RPEM algorithms since these can not be, necessarily, always working. Figures 1 and 2 represent the above described identification strategy.

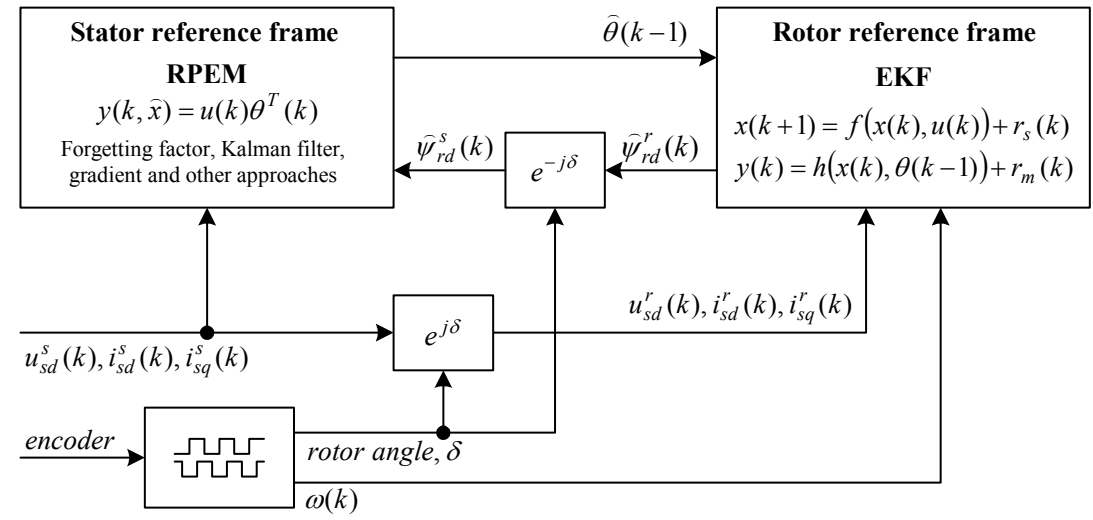

Fig. 1. The estimation methodology.

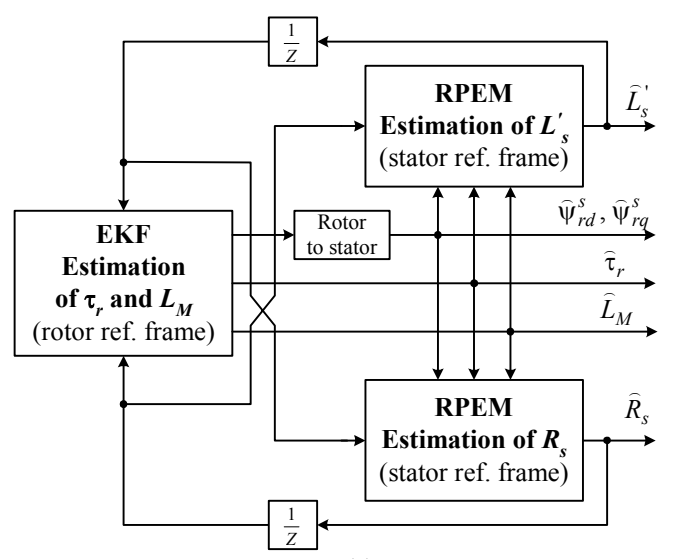

(a)

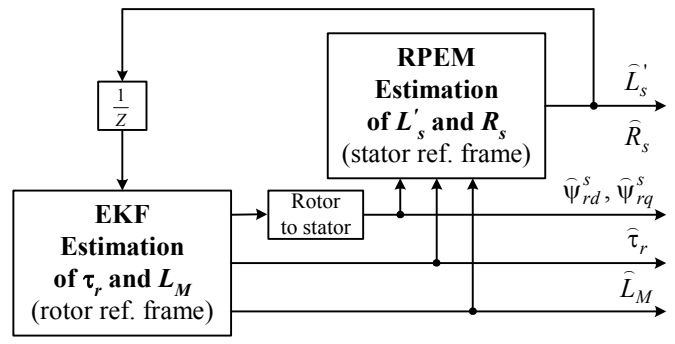

(b)

Fig. 2. The identification methodology with independent estimation of stator parameters and rotor states (parameters and flux components). (a) Separate estimation of stator parameters and (b) joint estimation of both stator parameters.

\section{Simulation Results}

The above-proposed estimator has been developed in the MATLAB with Simulink environment and tested under a vector control scheme. Simulation conditions were selected to be as close as possible of the experimental ones.
Fig. 3 shows the simulation results where we can see the rotor speed of a $2.2 \mathrm{~kW}$ vector controlled induction motor as well as the estimated flux and parameters, with the two stator parameters estimated by two recursive prediction error based estimators as shown in fig. 2(a) and the results of a validation test are also shown in figures $3(\mathrm{~g})$ and $3(\mathrm{~h})$. 


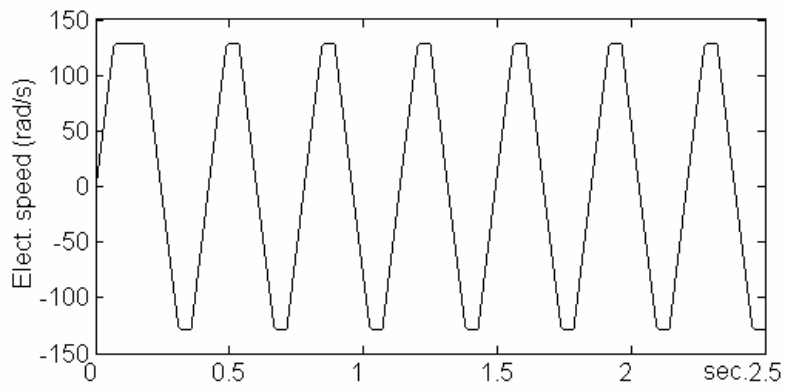

(a)

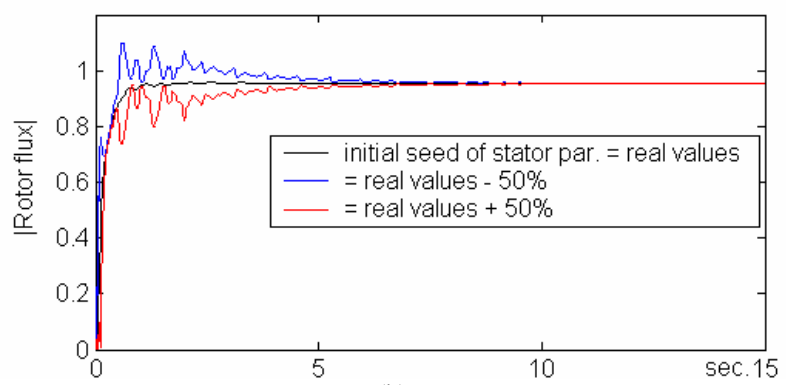

(b)

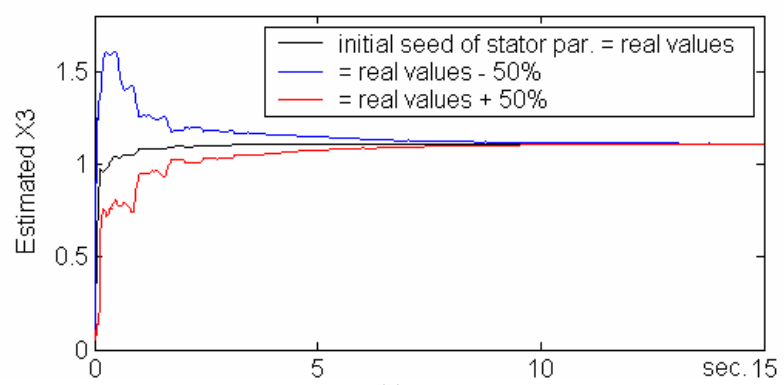

(c)

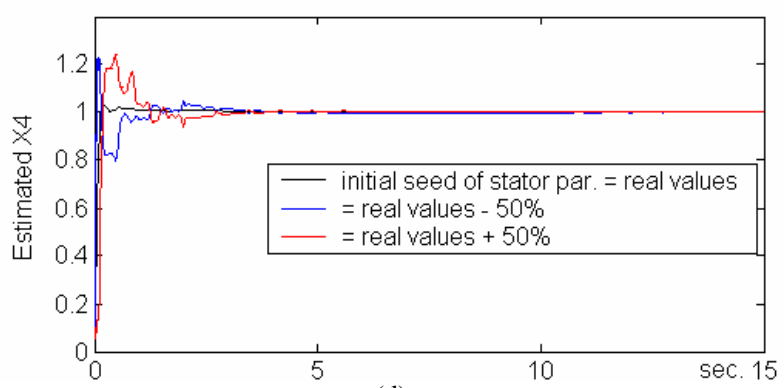

(d)

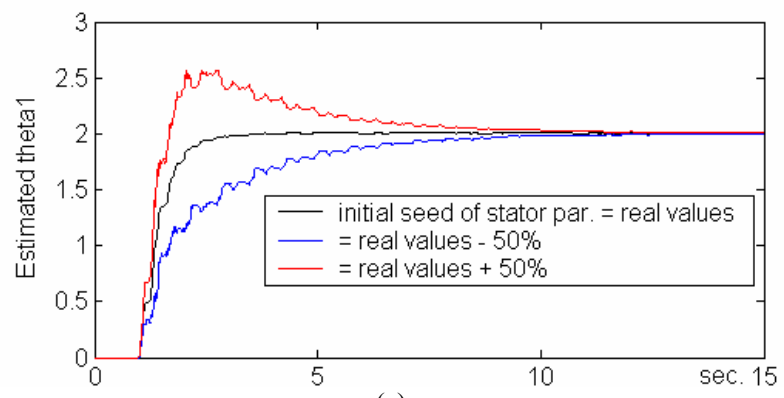

(e)

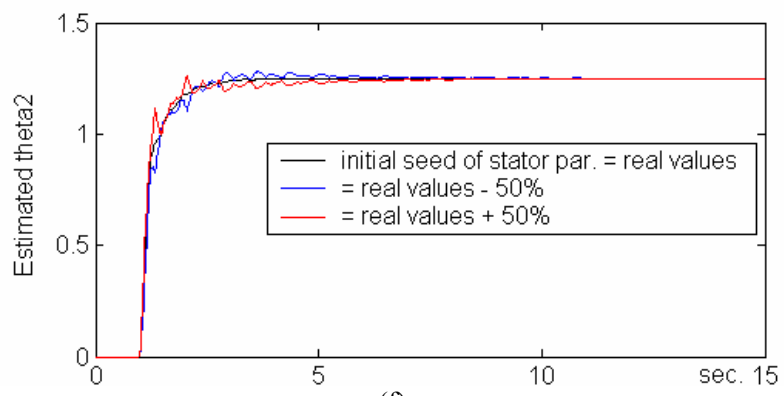

(f)

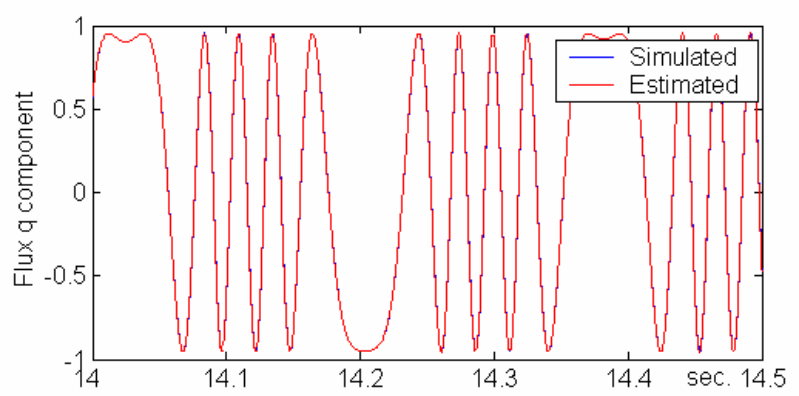

(g)

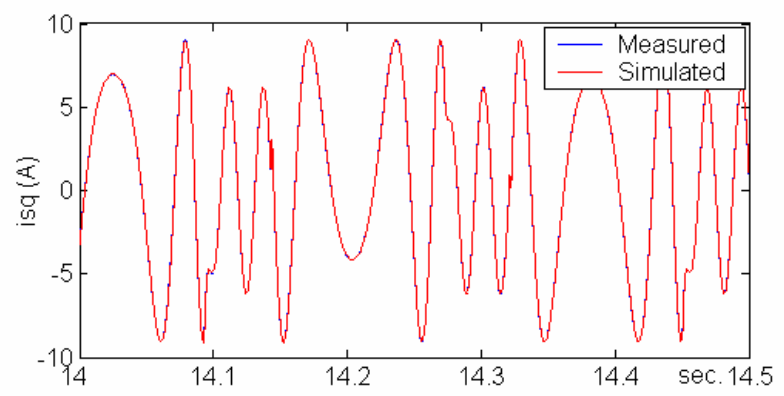

(h)

Fig. 3. Performance of the proposed identification methodology for different initial seeds of stator parameters used by the EKF. (a) Generated electrical rotor speed by the vector control scheme, (b) estimated magnitude of rotor

flux, (c) estimated state $x_{3}$ - scaled inverse of rotor time constant, (d) estimated state $x_{4}$ - scaled magnetizing inductance, (e) estimated parameter $\theta_{1}$ - scaled stator transient inductance, (f) estimated parameter $\theta_{2}$ - scaled stator resistance, (g) simulated/generated and estimated rotor flux $q$ component and

(h) generated stator current $q$ component and the simulated one using the online estimated parameters.

One of the main difficulties of any full parameter and flux estimator is the start-up procedure if no information is available beforehand about the electrical parameters. It is even not possible to do it, since we search for too much information as fluxes and parameters, based on just stator signals and rotor speed and, on the other hand, the model sensibility in relation to the stator parameters is low and strongly dependent of the dynamic conditions during the start-up and immediately after. Due to this, a robust solution is proposed here. We only need to have rough initial values of the electrical parameters for scaling the state vector $x$ in (3) and parameter vector $\theta$ in (4). Many solutions can be used for this purpose, some of them being very simple, such as 
classical methods or other rough parameter estimations as in [2], based on nominal characteristics of the induction motor.

Within the estimation algorithms themselves, the only requirement with respect to initial values of the electrical parameters is related to the initialization of stator parameters. Since in full estimation the rotor flux components are jointly estimated with the electrical parameters, the algorithms hardly converge to correct values because of the lack of confidence in estimated flux components at the beginning and, on the other hand, the lack of sensibility of the induction motor model with respect to stator parameters without specific supply conditions.

By this way, the EKF is started without any assumed knowledge of rotor parameters, but with initial seeds for stator parameters. Thus, 1 second after the EKF has been started, when the rotor parameters and flux are supposed to be close to the right values, the RPEM algorithm(s) is(are) started with rough estimates or even zero values in the parameter vector $\theta$ as used in (13). One second later all parameters and flux components are supposed to be converging to their real values and the algorithms start working according to the proposed identification methodology.

The initial values used in the algorithms are now presented. For the EKF, the initial state, the state covariance matrix and the system and measurement noise covariance matrices are, respectively:

$$
\begin{aligned}
& R_{s}(0)=R_{s}-50 \% ; \quad L_{s}^{\prime}(0)=L_{s}^{\prime}-50 \% \\
& x(0)=\left[\begin{array}{llll}
0 & 0 & 0.1 & 0.1
\end{array}\right] \\
& P(0)=\operatorname{diag}\left[\begin{array}{llll}
1 e-5 & 1 e-5 & 1 e-4 & 1 e-4
\end{array}\right] \\
& R_{s}=\operatorname{diag}\left[\begin{array}{llll}
1 e-8 & 1 e-8 & 1 e-9 & 1 e-9
\end{array}\right] \\
& R_{m}=10
\end{aligned}
$$

The RPEM algorithms were initialized as follows:

$$
\begin{array}{ll}
\theta_{1}(0)=0 & \theta_{2}(0)=0 \\
P_{\theta_{1}}(0)=1 e-3 & P_{\theta_{2}}(0)=1 e-3 \\
R_{s, \theta_{1}}=1 e-7 & R_{s, \theta_{2}}=1 e-8 \\
R_{m, \theta_{1}}=10 & R_{m, \theta_{2}}=10
\end{array}
$$

and they were based on the Kalman filter approach but other approaches can be used like forgetting factor (recursive least squares), normalized and unnormalized gradient approaches among other recursive prediction error methods that can be found in [8] and [9].

The scaling factors $k_{1}$ to $k_{6}$ referred in (3) and (4) were, respectively, $1,1,0.2,5,100$ and 0.5 .

The simulation results shown in fig. 3 were obtained with the configuration of fig. 2(a) and a square speed reference in the range -600 to $+600 \mathrm{rpm}$, in order to ensure persistent enough excitation of the stator transient inductance from the transient conditions, and stator resistance from the low speed zones, where the term $R_{s} i_{s}$ becomes important in the virtual measured output of the output equation expressed by (6).

In order to prove the robustness of the proposed methodology with respect to initial seeds of stator parameters in the EKF based algorithm, three runs were achieved with different initial seeds: In the first one the initial seeds were made equal to the parameters' real values and in the second and third they were made equal to $50 \%$ below and above of its real values, respectively. The results are presented in figures 3(b) to 3(f). The steady-state errors in all estimated parameters are less than $2 \%$, and the estimated flux waveform very closely matches the simulated one as shown in fig. $3(\mathrm{~g})$. Also the simulated stator current matches the "measured" one (generated by the vector control scheme) using the online estimated parameters, as can be seen in fig. 3(h).

\section{EXPERIMENTAL RESULTS}

Experimental results, shown in fig. 4, proved the feasibility and applicability of the methodology described in the previous sections, and the results of a practical experiment were recorded to validate the proposed strategy, and confirm the simulation results presented in the above section. In the practical experiment the stator voltages and stator currents, expressed in the stator reference frame, have been sampled at $5 \mathrm{kHz}$. Elliptic low-pass pre-filters of fifth order with a $500 \mathrm{~Hz}$ cutoff frequency have been used on the samples. Specific hardware was developed that has the signals available in the range of $\pm 10 \mathrm{~V}$ in both rotor and stator reference frames, by using the AD2S100 analogue processor, but only the stator-referred signals are used for this work. The stator voltages and currents are acquired in the stator reference frame with a data acquisition system that consists of the dSPACE development system, ACE Kit 1103, based on the DS1103 PPC controller board, the Real Time Interface (RTI) blockset for Simulink as well as experiment software (ControlDesk, MLIB/MTRACE). The dSPACE development platform was used for the real time identification task and not for control.

The rotor position and speed, needed for reference frame conversions, are obtained via an incremental encoder. For the EKF the stator voltages and currents are converted into the rotor reference frame and the estimated rotor flux $d q$ components are converted into the stator reference frame. Digital filters, with the same specifications as the analog ones, were used for filtering the computed rotor speed from rotor angle as well as electrical signals after being acquired. Unfortunately, both filters introduce delays. In order to synchronize all the signals, the rotor angle and filtered speed had to be suitably delayed. A $2.2 \mathrm{KW}$ induction motor controlled by an industrial frequency converter from $A B B$ has been used. The motor was loaded by a powder break which was programmed for $12 \mathrm{Nm}$, about half of the nominal torque.

The vector control scheme developed by us in Simulink 
used in the simulation experiment and the one implemented by the $A B B$ converter are naturally different. On the other hand, the electrical parameters are not constants anymore. By tuning the system and noise covariance matrices diagonal values, the gains in the identification algorithms may be adjusted either in order to track the respective electrical parameters following closer the instantaneous variations of the real parameters or, instead, to track the mean values over the time. Therefore some non-similarities exist between the generated signals in Simulink and the ones generated by the $A B B$ frequency converter and, consequently, the evolutions of the estimated parameters in these two phases of the work are a little bit different.

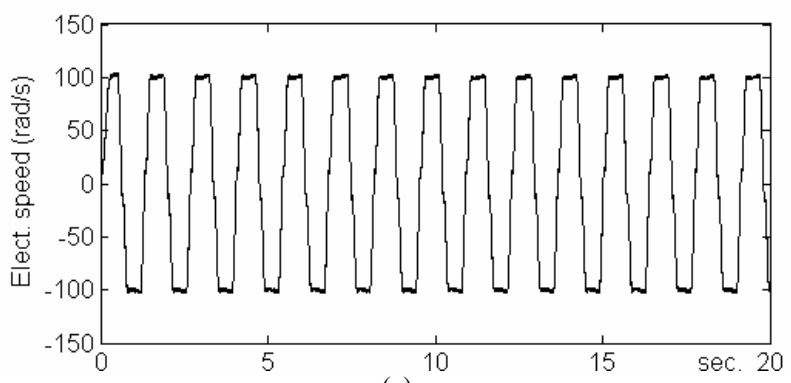

(a)
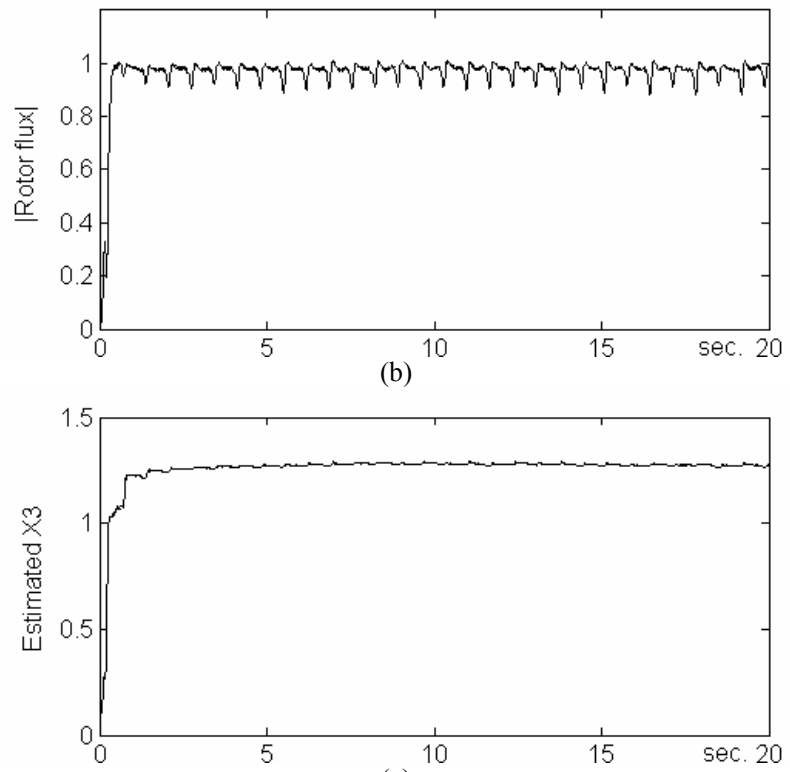

(c)

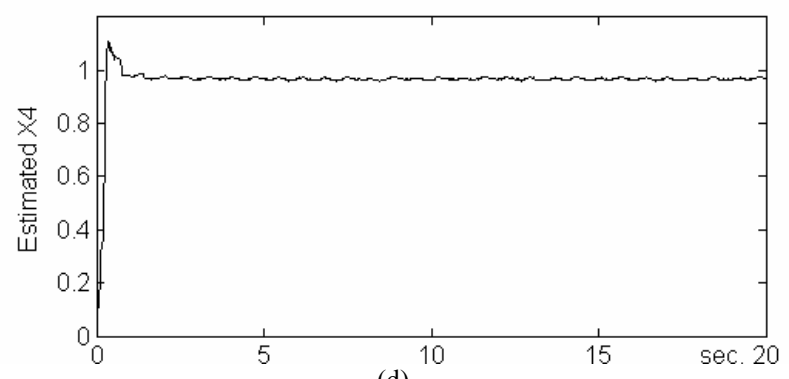

(d)

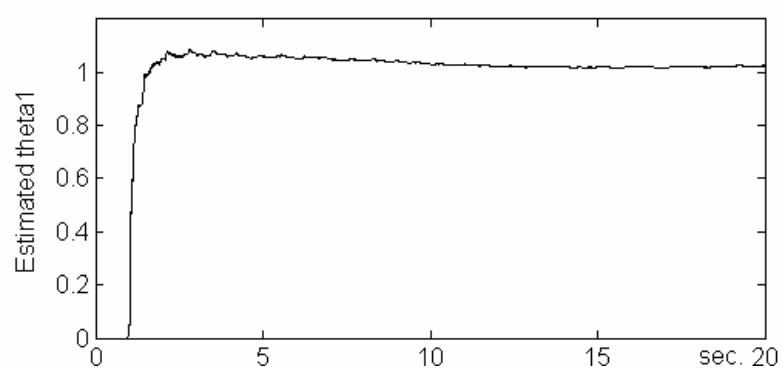

(e)
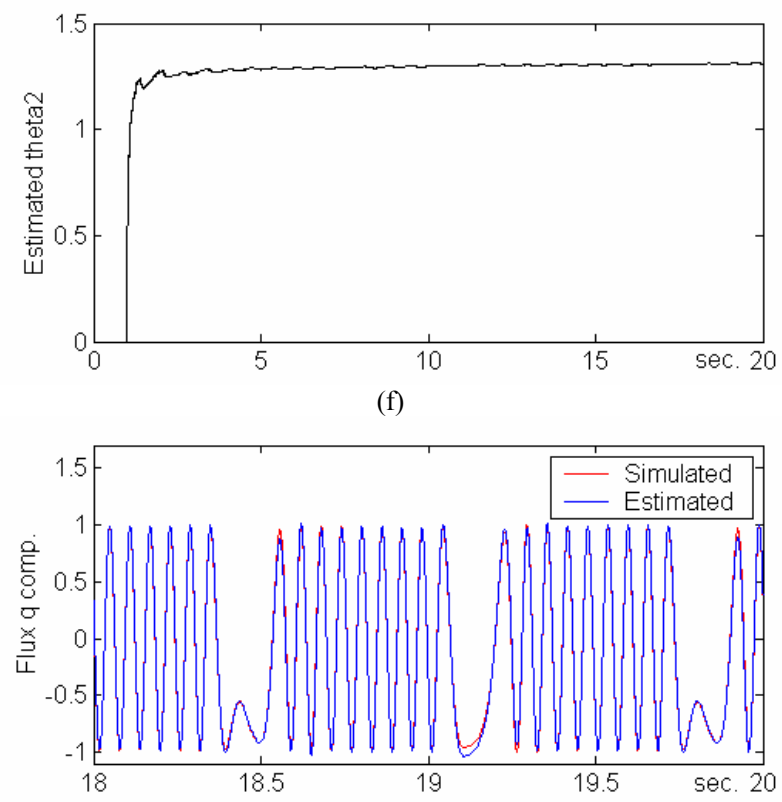

(g)

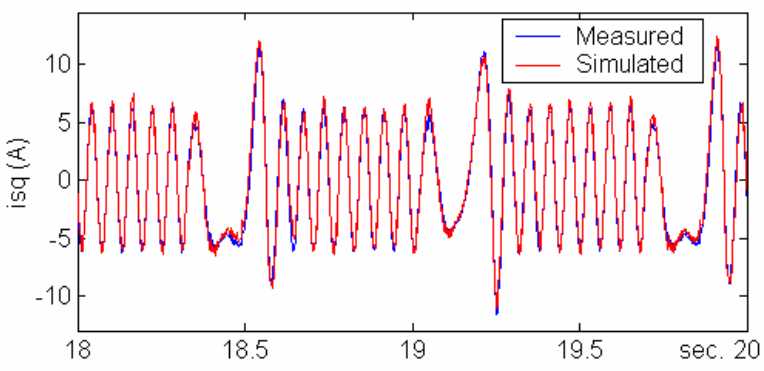

(h)

Fig. 4. Performance of the proposed identification methodology. (a) Measured electrical rotor speed, (b) estimated magnitude of rotor flux, (c) estimated state $x_{3}$ - scaled inverse of rotor time constant, (d) estimated state $x_{4}$ - scaled magnetizing inductance, (e) estimated parameter $\theta_{1}$ - stator scaled transient inductance, (f) estimated parameter $\theta_{2}$ - scaled stator resistance, (g) estimated and simulated rotor flux $q$ component and (h) generated stator current $q$ component and the simulated one using the online estimated parameters.

The experimental results, presented in fig. 4, show the performance of stator parameters estimation by using the recursive prediction error based approach and the rotor flux components and rotor parameters estimation by using the EKF.

In order to guarantee the validity of the identification 
methodology it is necessary to validate the identified induction motor model through some validation test to evaluate the performance of the algorithms. The validation test was based on the simulation of a modified induction motor model, with the online estimated parameters, by injection of the measured voltages and angular speed and subsequent comparison of simulated and measured stator current $d q$ components.

As can be seen, in fig. 4(g), the estimated flux, after converted into the stator reference frame, and the same one, simulated in the stator reference frame with the parameters estimated by the above-proposed methodology, are very similar. Like the rotor flux, the measured stator current, in the stator reference frame, closely matches the same current simulated with the estimated parameters, as shown in fig. 4(h).

\section{CONCLUSION}

The presented identification methodology, based on the above-proposed recursive estimators, is proposed for joint online flux and all electrical parameters estimation of a vector controlled induction motor by means of the EKF for rotor flux and rotor parameters, with independent estimation of the stator parameters by using a recursive prediction error based approach, instead of a full parameter and flux estimator with a high order EKF. It is not restricted to steady-state operation, being capable to operate in transient conditions and the possibility of enabling or disabling the stator parameters estimation becomes available, taking into account the momentary machine dynamics.

The stator and rotor reference frames are used for, respectively, stator parameters and rotor states (flux and parameters) estimation, as being the most natural ones for each case.

The proposed methodology enables the estimation with the EKF of rotor flux and parameters, at every iteration, in both transient and steady-state operation with rough initial seeds for stator parameters. One or both stator parameters can then be estimated, or updated from time to time, whenever the dynamics of the induction motor is suitable for this purpose. The independency of the estimators is an advantage and is even mandatory since the normal operating conditions are not always generating persistent excitation for parameter identification. Since stator resistance has slow dynamics when compared with the other electrical parameters and can be measured or even estimated from time to time, then if its value is assumed to be known we can improve even more the robustness of the estimation of the other three parameters.

The identification methodology that has just been presented in this paper can be applied for auto-tuning and adaptive direct field-oriented induction motor control.

\section{APPENDIX}

The EKF algorithm

$$
\begin{aligned}
& E\left\{x_{e}(0)\right\}=\hat{x}_{e}(0)=\left[\begin{array}{ll}
\hat{x}(k) & \hat{\theta}(k)
\end{array}\right] \quad E\left\{\left(x_{e}(0)-\hat{x}_{e}(0)\right)\left(x_{e}(0)-\hat{x}_{e}(0)\right)^{T}\right\}=P(0) \\
& \hat{x}_{e}(k+1 \mid k)=\left[\begin{array}{c}
f(\hat{x}(k \mid k), u(k), \hat{\theta}(k \mid k)) \\
\theta(k)
\end{array}\right]+\left[\begin{array}{l}
r_{s}(k) \\
r_{\theta}(k)
\end{array}\right]=f_{e}\left(\hat{x}_{e}(k \mid k), u(k)\right)+r_{s e}(k) \\
& F(k)=\left.\frac{\partial f_{e}\left(x_{e}(k), u(k)\right)}{\partial x_{e}^{T}(k)}\right|_{x_{e}(k)=\bar{x}_{e}(k \mid k)}=\left[\left.\begin{array}{cc}
\frac{\partial f_{e}(x(k), u(k), \theta(k))}{\partial x^{T}(k)} & \frac{\partial f_{e}(x(k), u(k), \theta(k))}{\partial \theta^{T}(k)} \\
I
\end{array}\right|_{\hat{x}_{e}(k \mid k)}\right.
\end{aligned}
$$

$\hat{P}(k+1 \mid k)=F(k) \hat{P}(k \mid k) F^{T}(k)+R_{s}$

$$
\begin{aligned}
& H(k)=\left.\frac{\partial h_{e}\left(x_{e}(k)\right)}{\partial x_{e}^{T}(k)}\right|_{\hat{x}_{e}(k+1 \mid k)}=\left[\left.\frac{\partial h(x(k), \theta(k))}{\partial x^{T}(k)} \frac{\partial h(x(k), \theta(k))}{\partial \theta^{T}(k)}\right|_{\hat{x}_{e}(k+1 \mid k)}\right. \\
& K(k+1)=\hat{P}(k+1 \mid k) H^{T}(k)\left[H(k) \hat{P}(k+1 \mid k) H^{T}(k)+R_{m}\right]^{-1} \\
& \hat{y}(k+1)=h\left(\hat{x}_{e}(k+1 \mid k), k\right) \\
& \widehat{x}_{e}(k+1 \mid k+1)=\hat{x}_{e}(k+1 \mid k)+K(k+1)[y(k+1)-\hat{y}(k+1)] \\
& \hat{P}(k+1 \mid k+1)=[I-K(k+1) H(k)] \hat{P}(k+1 \mid k)
\end{aligned}
$$

The RPEM algorithm

$$
\begin{aligned}
& \psi(k+1)=\partial \widehat{y}(k, \theta) /\left.\partial \theta\right|_{\theta=\hat{\theta}(k)}, \quad \psi-\text { gradient } \\
& L(k+1)=P(k) \psi(k+1)\left[\psi^{T}(k+1) P(k) \psi(k+1)+R_{m}\right]^{-1} \\
& \hat{\theta}(k+1)=\hat{\theta}(k)+L(k+1)(y(k+1)-\hat{y}(k+1)) \\
& P(k+1)=P(k)-L(k+1) \psi^{T}(k+1) P(k)+R_{s}
\end{aligned}
$$

\section{ACKNOWLEDGMENT}

The authors would like to thank the availability of the laboratorial resources by the Laboratório de Electrónica e Instrumentação during the preparation of this paper.

\section{REFERENCES}

[1] T. Du, P. Vas, F. Stronach, "Design and Application of Extended Observers for Joint State and Parameter Estimation in HighPerformance AC Drives", IEE Electr. Power Appl., vol. 142, n. ${ }^{\circ}$ 2, pp. 71-78, March 1995.

[2] L. Loron., G. Laliberté, "Application of the Extended Kalman Filter to Parameters Estimation of Induction Motors", in Proc. EPE, Brighton, 1993 pp. 85-90.

[3] J. W. Finch, D. J. Atkinson, P. P. Acarnley, "Full-Order Estimator for Induction Motor States and Parameters", IEE Electr. Power Appl., vol. 145, n. ${ }^{\circ} 3$, pp. 169-179, May 1998.

[4] V. Leite, R. Araújo, D. Freitas, "Flux and Parameters Identification of Vector-Controlled Induction Motor in the Rotor Reference Frame", in Proc. $A M C^{\prime} 02$, Maribor, Slovenia, July 4-5, 2002, pp. 263-268.

[5] V. Leite, R. Araújo, D. Freitas, "A Real-time Estimator of Electrical Parameters for Vector Controlled Induction Motor using a Reduced Order Extended Kalman Filter", in Proc. EPE-PEMC'02, Cavtat \& Dubrovnik, Croatia, September 9-11, 2002, paper T11-029.

[6] P. Vas, "Parameter Estimation, Condition Monitoring, and Diagnosis of Electrical Machines", Oxford Science Publications, 1993.

[7] A. J. L. Harrison, D. P. Stoten, "Generalized Finite Difference Methods for Optimal Estimation of Derivatives in real-Time Control Problems", in Proc. Instn Mech Engrs, vol. 209, pp 67-78, 1995.

[8] G. C. Goodwin, K. S. Sin, "Adaptive Filtering Prediction and Control", Prentice-Hall, 1984.

[9] L. Ljung, "System Identification, Theory for the User", Prentice Hall, 1999. 